\title{
Exploring cooperation and competition in the Centipede game through verbal protocol analysis
}

\author{
Eva M. Krockow*, Andrew M. Colman, and Briony D. Pulford \\ School of Psychology, University of Leicester, UK \\ *Correspondence should be addressed to Eva M. Krockow, School of Psychology, University of Leicester, \\ Leicester LE1 7RH, United Kingdom, (e-mail: emk12@le.ac.uk).
}

Accepted for publication in the European Journal of Social Psychology (2016)

\section{Acknowledgements}

The research reported in this article was supported by an award from the Friedrich-Naumann-Stiftung für die Freiheit. The authors are grateful to Diana Pinto for help with data collection and to Kevin McCracken for help with software development.

\begin{abstract}
The Centipede game is an abstract model of reciprocal relationships where two individuals alternate in helping each other at relatively small personal cost. Whereas mutual cooperation can benefit both individuals in the long run, a paradoxical but logically compelling backward induction argument shows that cooperation is irrational. Empirical studies have reported reliable deviations from the non-cooperative backward induction solution, but their exclusively quantitative methods allow only a limited range of predefined motives to be explored. Our study uses verbal ('think aloud') protocols and qualitative data analysis to identify motives for cooperation in the Centipede game. The results provide little evidence for sophisticated backward induction reasoning. Instead, a wide range of motives emerged, their relative saliences varying according to the stage of the game. Activity bias affected decisions mainly at the beginning of the game, whereas cooperative and altruistic social value orientations most frequently accounted for cooperation towards its natural end.
\end{abstract}

Keywords: Centipede game; verbal protocols; Social value orientation; cooperation; Theory of Mind

Most prolonged human relationships rely on a reciprocal give-and-take of time and other resources. In a romantic relationship, two partners may take turns comforting each other after a long day at work; in a friendship, two mates may alternate in hosting football evenings at their homes; and in a professional work relationship, two colleagues may support each other's projects during particularly stressful periods. All these relationships share a basic structure of alternating cooperation (providing comfort, hosting football parties, or offering work support) that is costly to the co-operator in time and energy but yields important benefits to the other person (relaxation, fun, or occupational success) and carries the possibility of having the favour returned in the future.

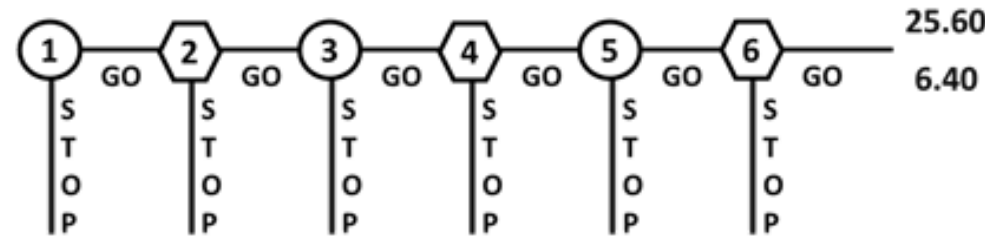

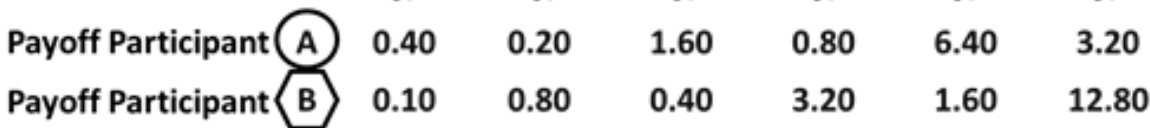

Figure 1. Exponentially increasing Centipede game adapted from McKelvey and Palfrey (1992). 
Interactions of reciprocal cooperation such as these can be modelled by Rosenthal's (1981) Centipede game (Figure 1), which represents the underlying strategic structure of reciprocal relationships. The interaction proceeds from left to right in the diagram across the six numbered decision nodes. Two decision makers, Player A and Player B, alternate in choosing between a cooperative GO move, which keeps the interaction going, and a noncooperative or defecting STOP move, which leads to an exit node and terminates the interaction. Each decision in the Centipede game is associated with a numerical payoff displayed at the bottom of the diagram. The payoff units could represent sweets or marbles or any material or immaterial benefits whatsoever, but for simplicity we shall assume that the units are pounds sterling. Since cooperative actions are costly in the real world, each analogous GO move in the Centipede game leads to a decrease of the individual's payoff - in the example game, the personal payoff is halved. Simultaneously, however, it benefits the coplayer by multiplying that player's payoff eightfold, and doubles the pot of combined payoffs to the player pair. Hence, if the two players engage in reciprocal GO moves, they benefit in the long run. The numbers in the diagram are the cumulative payoffs, realised only if the game is stopped at the each of the associated decision nodes.

Nevertheless - and surprisingly - a mathematical analysis of the Centipede game based on backward induction (BI) reasoning dictates immediate defection at the first decision node as the only rational outcome of the game (Aumann 1995, 1996). The BI argument starts at the final decision node (in this case, Node 6) where it is Player B's turn to move. A GO move would lead to the game's natural end with a personal payoff of $£ 6.40$ whereas a STOP move would terminate the interaction prematurely with a payoff of $£ 12.80$. Any Player B who is instrumentally rational in the sense of aiming to maximise personal gain will choose a STOP move at Node 6, because cooperating at that last decision node yields a smaller payoff to that player. Player A, when making a decision at Node 5, should foresee that a GO move would be followed by Player B's defection immediately after, yielding a modest $£ 3.20$ to Player A, whereas a STOP move would end the game with twice as much personal gain (£6.40). Provided that both players are instrumentally rational and that this, and the specification of the game including the payoffs are common knowledge in the game (in the sense that both players know them, both know that both know them, and so on), the argument can be extended backwards by another step, to Node 4, where Player B has to make a choice. A GO move would, as already shown, lead to Player A terminating the game at Node 5, resulting in a disappointing payoff of $£ 1.60$ to Player B, whereas a STOP move at Node 4 would end the game with a comparatively better payoff of $£ 3.20$. This so-called stepwise elimination of dominated strategies can be continued to the very beginning of the game, where it dictates immediate defection by Player A, yielding a minimal payoff of $£ 0.40$.

This game-theoretic solution (the subgame perfect Nash equilibrium of the game), despite appearing logically compelling, is difficult or impossible for any decision maker with common sense to accept. The Centipede game presented in Figure 1 tempts Player A with a maximum payoff of $£ 25.60$ on the right. How can it be instrumentally rational (by definition, payoff-maximizing) to defect at Node 1 and content oneself with a negligible 40p? Empirical studies of the Centipede game confirm that human decision makers rarely act according to the game-theoretic solution; most cooperate for at least a few decision nodes, and some even choose GO moves at the final decision node, thus irrevocably reducing their own payoff but benefitting the other person and the overall payoff to the player pair (e.g., Bornstein, Kugler, \& Ziegelmeyer, 2004; McKelvey \& Palfrey, 1992; Nagel \& Tang, 1998).

Different explanations have been suggested for the pervasive deviations from the game-theoretic solution. Generally, these explanations can be divided into two strands, focusing either on insufficient cognitive ability to perform BI reasoning, or on other- 
regarding motives that might interfere with rational (but selfish) decision making. We shall now outline the most prominent and influential theory associated with each of these strands.

\section{Theory of Mind}

Theory of Mind (ToM) is a psychological theory describing the cognitive processes involved in perspective-taking to infer another person's knowledge, beliefs, and intentions. Whereas the bulk of research on ToM has been conducted by developmental psychologists examining children's awareness of other people's mental states, it has more recently been applied to strategic games in order to investigate the sophistication of reasoning levels by human decision makers (Doshi, Qu, Goodie, \& Young, 2012; Goodie, Doshi, \& Young, 2012; Hedden \& Zhang, 2002; Zhang, Hedden, \& Chia, 2012). According to ToM, the players' predictions about their co-players' preferences and strategies in the game influence their own choices. Accurate assumptions about the other person's future moves make it possible to identify best replies that maximise personal payoffs. In the Centipede game, the best reply to a co-player's strategy of stopping at Node $x_{n}$ is invariably to exit at the immediately preceding Node $x_{n-1}$ (Droste, Kosfeld, \& Voorneveld, 2003). ToM categorises decision makers according to the number of steps of recursive perspective-taking they can perform, and these are referred to as levels of rationality (e.g., Goodie, Doshi, \& Young, 2012): 0thlevel rationality is assigned to individuals, who make decisions based on their personal preferences without consideration of their fellow decision makers' likely moves. In the Centipede game, a Player A with 0th-level rationality is likely to identify the highest possible personal outcome (i.e., £25.50 at Exit Node 7) and aim for that outcome without taking into account Player B's likely STOP move at Node 6. The next higher level of reasoning, 1st-level rationality, refers to individuals who act to maximise their personal payoffs on the assumption that their co-players are 0th-level reasoners. A Player A with 1st-level reasoning would be able to predict Player B's exit move at the penultimate node, and would consequently chose to exit the game at the preceding Node 5. The next higher level of reasoning, 2nd-level rationality, applies to individuals attributing 1st- and 0th-level rationality to the co-players, and so forth. With every additional level of rationality, the individual is able to perform one more step of iterated reasoning. To achieve the perfect BI outcome in a 6-node version of the Centipede game, Player A would thus require 3rd-level rationality.

Early experiments assessing the frequencies of different reasoning types used $3 \times 3$ matrix games and the 'Guess the Average Number' game. Results showed that most players initially exhibited 1st- or 2nd-level reasoning and improved their performance slightly with experience (Nagel, 1995; Stahl \& Wilson, 1995). In the context of the Centipede game, several investigators have proposed econometric models based on the closely related economic counterparts of ToM; Cognitive Hierarchy Theory and Level- $k$ reasoning (e.g., Camerer, Ho, \& Chong, 2004; Ho \& Su, 2011; Kawagoe \& Takizawa, 2012). More recent research (e.g., Colman, Pulford, \& Lawrence, 2014; Hedden \& Zhang, 2002) has confirmed that 1st-level reasoning is most common, followed by 2 nd-level reasoning.

A study by Palacios-Huerta and Volij (2009) found convergence towards the gametheoretic solution in a sample of expert chess players, who were assumed to have very high levels of iterated reasoning ability. However, cooperation levels among the chess players increased when they were paired with university students of supposedly lesser strategic reasoning ability. Taken together, these findings indicate that recursive reasoning skills and assumptions about co-players' cognitive capacities could have significant effects on decision making in the Centipede game.

Nevertheless, none of these studies directly assessed reasoning ability or explored alternative motives for move choices in the game. Also, the findings by Palacios-Huerta and Volij (2009) were challenged by a replication in which chess grandmasters made very similar 
decisions in the Centipede game to student samples (Levitt, List, \& Sadoff, 2011). Finally, the Centipede game, whose mixed-motive payoff function allows for social gains to the player pair, may elicit a wider range of motives than the previously studied Guess the Average Number game with its strictly competitive (zero-sum) payoff function, in which there is no scope for social gains and hence no incentive to cooperate.

\section{Social value orientations (SVO)}

Many different other-regarding motives could potentially explain cooperation in the Centipede game. The most frequently suggested include altruistic and team-orientated payoff preferences. Those and related preferences regarding the distribution of monetary resources between oneself and others are conveniently interpreted within the psychological framework of social value orientation, a concept introduced by Messick and McClintock (1968) and McClintock (1972). Initially, the concept included only cooperative, individualistic, and competitive orientations, but it was later extended to cover altruistic and equality-seeking preferences as well (Kelley \& Thibaut, 1978; Van Lange, 1999). In the context of two-player experimental games, individuals with different orientations can be characterised as follows: Cooperative players seek to maximise the combined payoffs of the player pair; individualistic players seek to maximise their personal payoffs; competitive players seek to maximise the difference between their personal payoffs and their co-players' payoffs; altruistic players seek to maximise their co-players' payoffs; and equality-seeking players aim for an even split of the payoffs between themselves and their co-players.

SVO has been shown to affect levels of cooperation in experimental games (see Balliet, Parks, \& Joireman, 2009, for a review), and a recent study by Pulford, Krockow, Colman and Lawrence (2015) found that both state SVO (induced by task framing instructions) and trait SVO (assessed by a questionnaire measure) significantly affected decision making in the Centipede game. Across two experiments, Pulford et al. showed that cooperative and individualistic state SVO led to significantly later exit moves (higher levels of cooperation) than a competitive state SVO. The influence of trait SVO depended on the state SVO that had been experimentally induced. So far, however, no research has investigated the effects of equality-seeking or altruistic SVO, the latter of which has frequently been suggested as an explanation of cooperation at the game's final decision node (e.g., McKelvey \& Palfrey, 1992).

\section{Qualitative research}

Previous research on the Centipede game has been dominated by quantitative studies. Based on the data obtained, various econometric models have been derived, but although these may provide more or less accurate predictions of decision making, their explanatory power is limited. McKelvey and Palfrey's (1992) initial model of their Centipede data, for example, contained a parameter to account for individuals who never defect in the game. The authors speculated that this player type may be altruistic, but this is an empirical assumption that is open to experimental testing. Hard-core cooperators could, for example, be driven by an action bias (enjoying the mere interaction and offering the other player a chance to participate); they may be acting in accordance with implicit demand characteristics; or their cooperative moves may be determined by misunderstandings of the instructions or the payoffs.

Existing models cannot capture the multitude of explanations underlying decision making in the Centipede game, and to advance our understanding of motives for cooperation in this game, analysis of qualitative data derived from vocal records of participants' reasoning processes during the game provides a promising avenue of investigation. We report the results of such an investigation in this article. 
Verbal protocol analysis. The generation of data from verbalisations of thoughts and feelings can yield a wealth of information on human cognitive and emotional processes. Concurrent verbalisation during task performance (rather than retrospective verbalisation after task completion) is likely to produce the best results in the context of experimental games, because the verbal records created during the task cannot be degraded by forgetting or hindsight biases. This method was previously criticised for its possible interference with task performance, but its influence on cognitive processes was demonstrated to be non-significant across several studies, provided that instructions to think aloud were kept general rather than targeted to yield specific contents such as explanations or reasons for behaviour (see Ericsson \& Simon, 1993, for a review). The only reliable influence of concurrent verbalisations was shown to be an increase of the time taken to complete a task.

Our study adopted an exploratory approach towards the investigation of decision making in the Centipede game. Using verbal protocols, we investigated strategic reasoning levels, social motivations (including SVOs), and other motives for choices in the game. We paid additional attention to the participants' processing of the task instructions and experimental cues that could suggest methodological improvements for future experiments.

\section{Method}

Participants. The sample comprised 12 students and members of staff from the University of Leicester (six males and six females) with a mean age of 26.33 years (SD = 6.59). They were incentivised with a $£ 5.00$ show-up fee and additional remuneration according to their payoffs in one round chosen randomly from the ten completed during the testing session. The mean remuneration per participant was $£ 12.08$, including the show-up fee.

Design. Six testing sessions were conducted with one pair of players in each. The computer randomly assigned the participants to the role of either Player A or Player B, and they stayed within that role for the duration of the testing session. In their pairs, they played ten rounds of the exponentially increasing Centipede game displayed in Figure 1 while verbalising their thoughts.

Materials. The study was conducted in two laboratories located in different buildings to ensure complete anonymity of the two participants interacting with one another. Each laboratory contained a computer with internet access, a digital voice recorder, and a video camera placed behind the participant. The video camera was used to back up the voice recording and to match the verbalisation with the display on the computer screen. For the interaction between the two participants, we used a custom-made, web-based game application that included several detailed instructions slides and a colour-coded, animated display of the Centipede game.

Procedure. Testing sessions lasted between 30 and 45 minutes. The two participants in each session were met in front of different buildings by two different experimenters and shown to the respective laboratories. Participants were informed about the study's procedure and made aware of the use of voice recorders and cameras. To practise their verbalisation skills, they were given a well-known logic puzzle - a four-disk, electronic version of the Tower of Hanoi-and they were asked to try to solve it while thinking aloud.

Following this training, participants received detailed on-screen instructions about the rules and payoff structure of the Centipede game, presented to them as a 'decision-making task' (words such as 'game', 'player' and 'cooperation' were avoided to minimise priming effects). The voice recorders and cameras were then switched on, and the participants interacted over ten rounds of the Centipede game while verbalising their thoughts. The exact instructions, based on Ericsson and Simon (1998), were 
Please remember to talk aloud for the whole duration of the decision sequence, just like you did in the previous training session. Verbalise everything that passes through your head whilst reaching a decision in the task. If you need to read something on the computer screen, please read aloud. Do not try to explain anything to anyone else. Pretend there is no one here but yourself.

Whenever participants stopped speaking, the experimenter prompted them as follows: 'Please keep talking' or 'Please verbalise your thoughts'.

Data analysis. For all games completed, the exit nodes, ranging between 1 and 7 , were recorded. Additionally, all verbal data were transcribed and analysed using NVivo Qualitative Data Analysis Software, Version 10 (2012). For each participant, a separate data file containing the verbal records was uploaded as a coding source in NVivo. Verbal statements were classified in a hierarchical network of 'parent' and 'child' coding nodes, with each coding node representing a different statement category and serving to collect references for that particular category. Whereas the initial network was theory-driven and centred on the two thematic pillars of depth of strategic reasoning and social motivation, it was adapted, refined, and extended though a bottom-up coding process. In its final form, the data also included coding nodes for study concerns (e.g., doubts about the reality of payoffs), the type of talk recorded (e.g., purely descriptive talk versus detailed deliberation about the game), and significant quotes. Finally, in order to investigate the salience of specific strategic reasoning levels and motives at specific decision nodes in the game, a contextual category was created containing coding nodes for the moves 'GO'; 'GO at first node'; 'GO at last node'; 'STOP' and 'STOP at first node'. A display of the most relevant coding categoriesreasoning level and other-regarding motives - including both parent and child coding nodes with example statements is displayed in Table 1.

\section{Results}

Quantitative results. The quantitative results are displayed in Table 2. The mean exit node across 60 rounds of the Centipede game was $5.12(S D=1.78)$. Only a single game was exited at the first decision node, and 18 games (30\%) reached their natural end without any defecting move. The observed patterns of quantitative game results for each player pair fell into one of three categories.

Perfect cooperation. The games completed by Pairs 1 and 3 showed almost perfect cooperation across all ten rounds of the game. Pair 1 deviated slightly from this pattern on the 9th and 10th rounds, stopping at Node 6 instead of Node 7, but the overall level of cooperation was almost fully cooperative.

Head-to-head. The games by Pairs 2 and 4 were characterised by fairly even numbers of STOP moves by the two players. For these two pairs, the ratios of STOP moves by Player A compared to Player B were 5:5 and 3:7 respectively. None of the games reached its natural end, and one of Pair 4's games was exited at the first decision node.

Dominant defector. The interactions by Pairs 5 and 6 showed a marked dominance by one player-in Pair 5, it was Player B, in Pair 6, Player A. The dominant players accounted for at least $90 \%$ of the exit moves across ten rounds, but their exit points varied; Pair 5 exited at decision nodes ranging between 2 and 6; Pair 6 exited at Node 5 in nine out of ten games.

Qualitative results. The number of verbal statements associated with each coding node are displayed in Table 1, and participant profiles, providing overviews of all motives and reasoning levels at the participant level, are shown in Table 3 with graphical illustrations displayed in Figures 2-7. Despite the verbalisation training, both quantity and quality of the verbalisations varied considerably from one participant to another. Participant 11 produced the most data (almost 1,500 words), and Participant 9 produced the least (less than 200 words). 


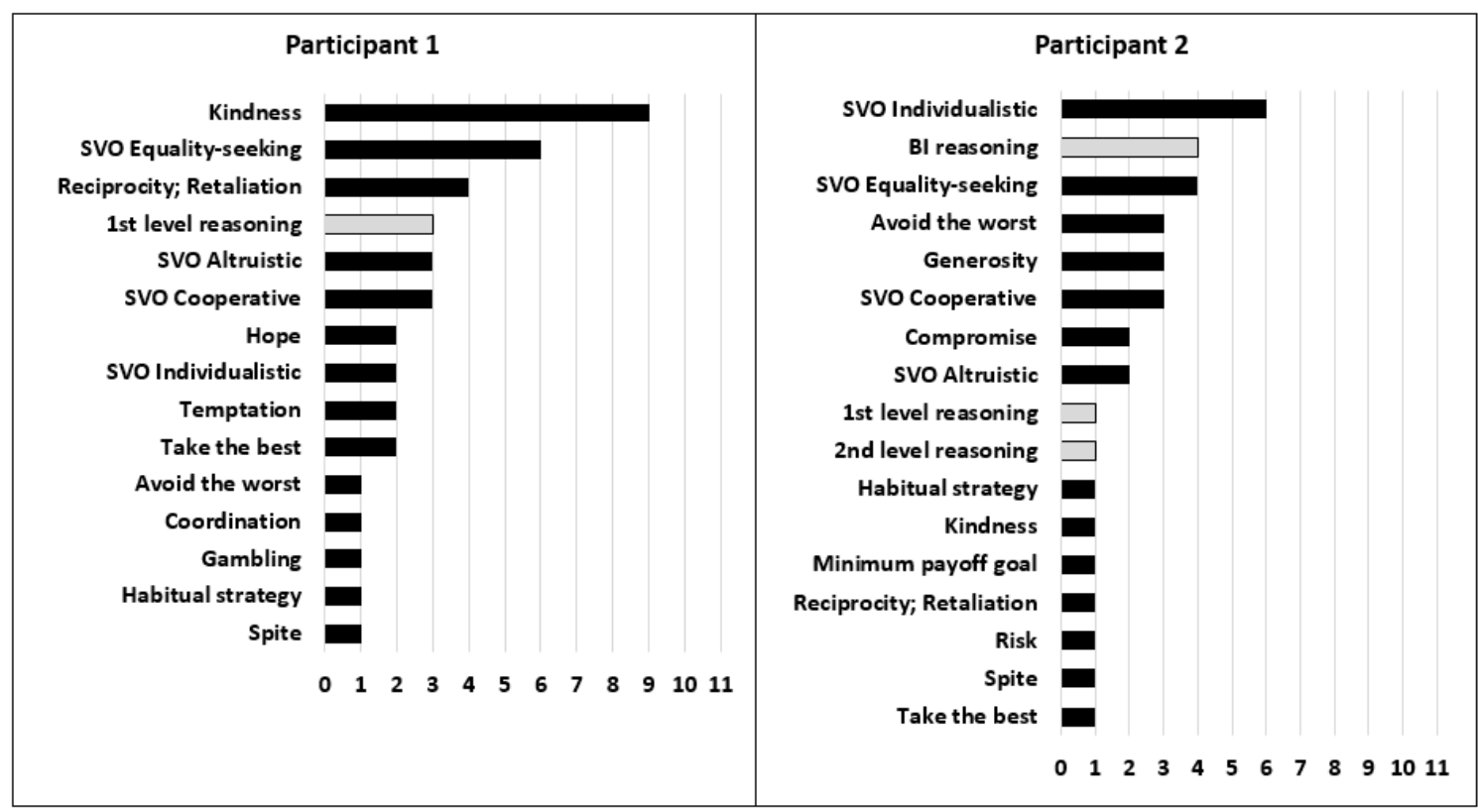

Figure 2. Number of coding references for Pair 1's levels of reasoning (grey bars) and motives (black bars).

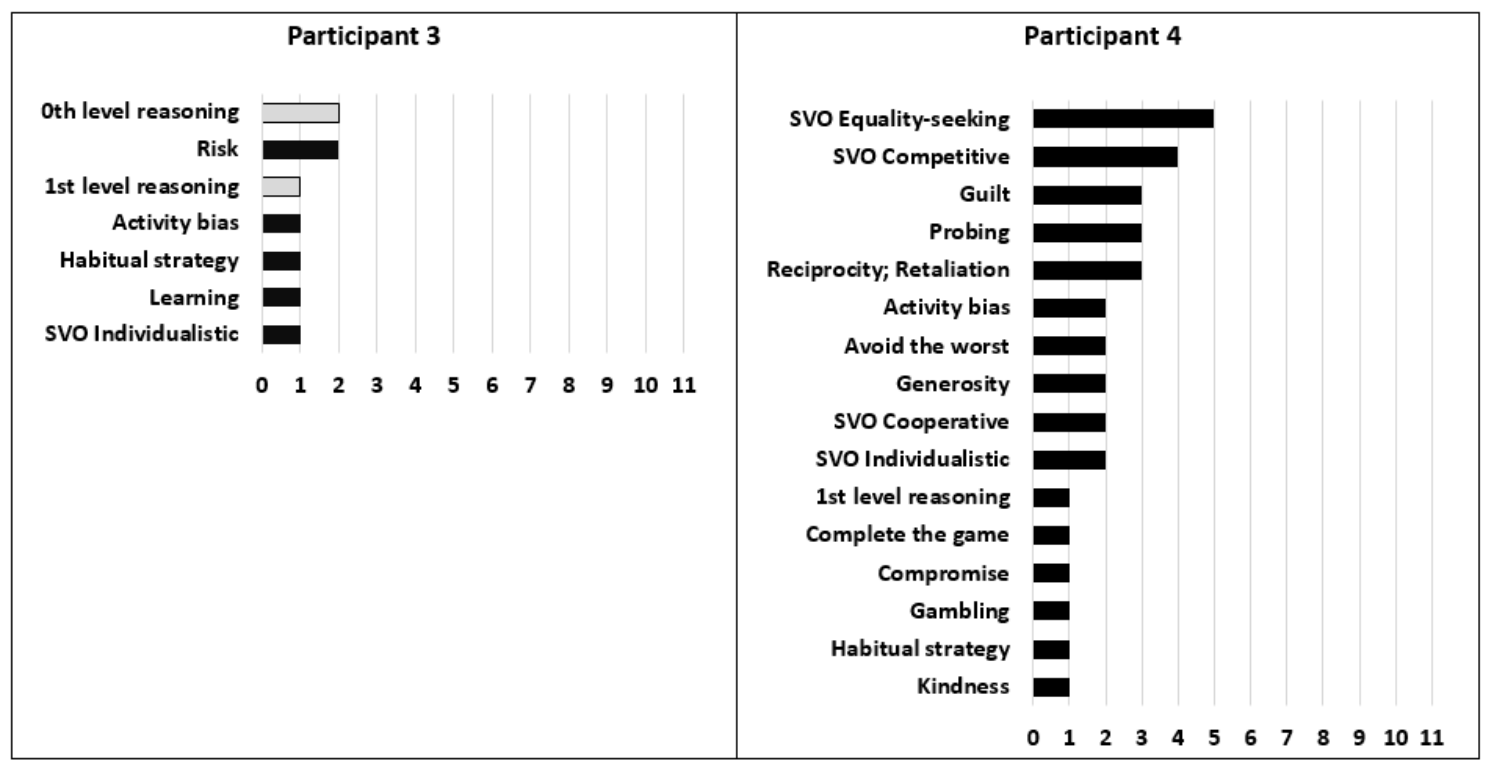

Figure 3. Number of coding references for Pair 2's levels of reasoning (grey bars) and motives (black bars).

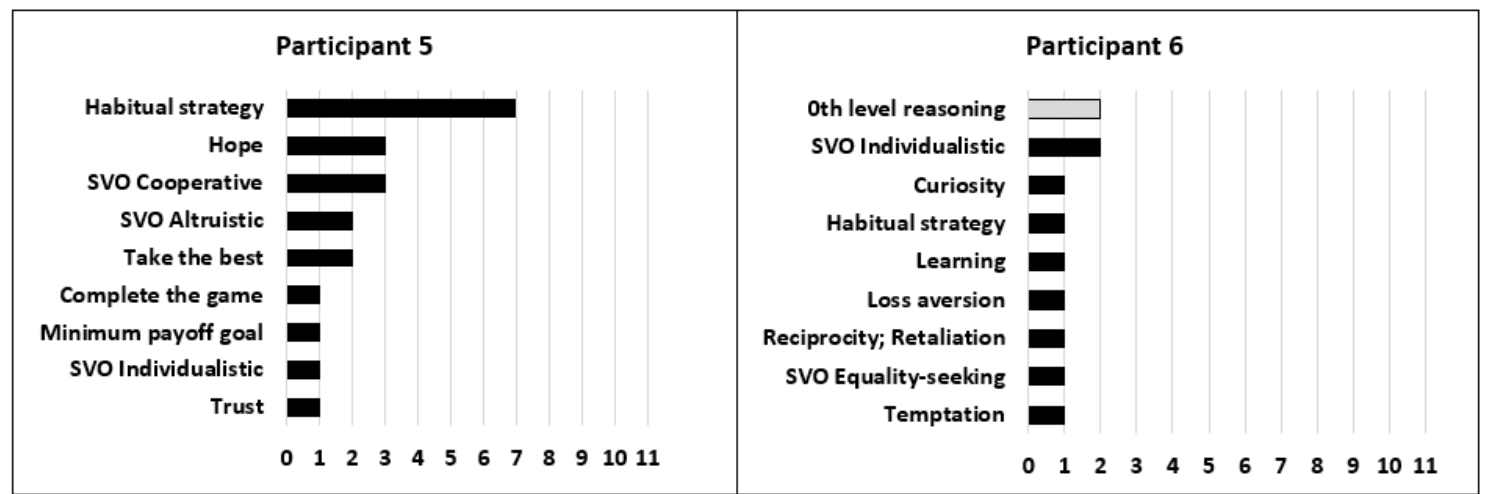

Figure 4. Number of coding references for Pair 3's levels of reasoning (grey bar) and motives (black bars). 


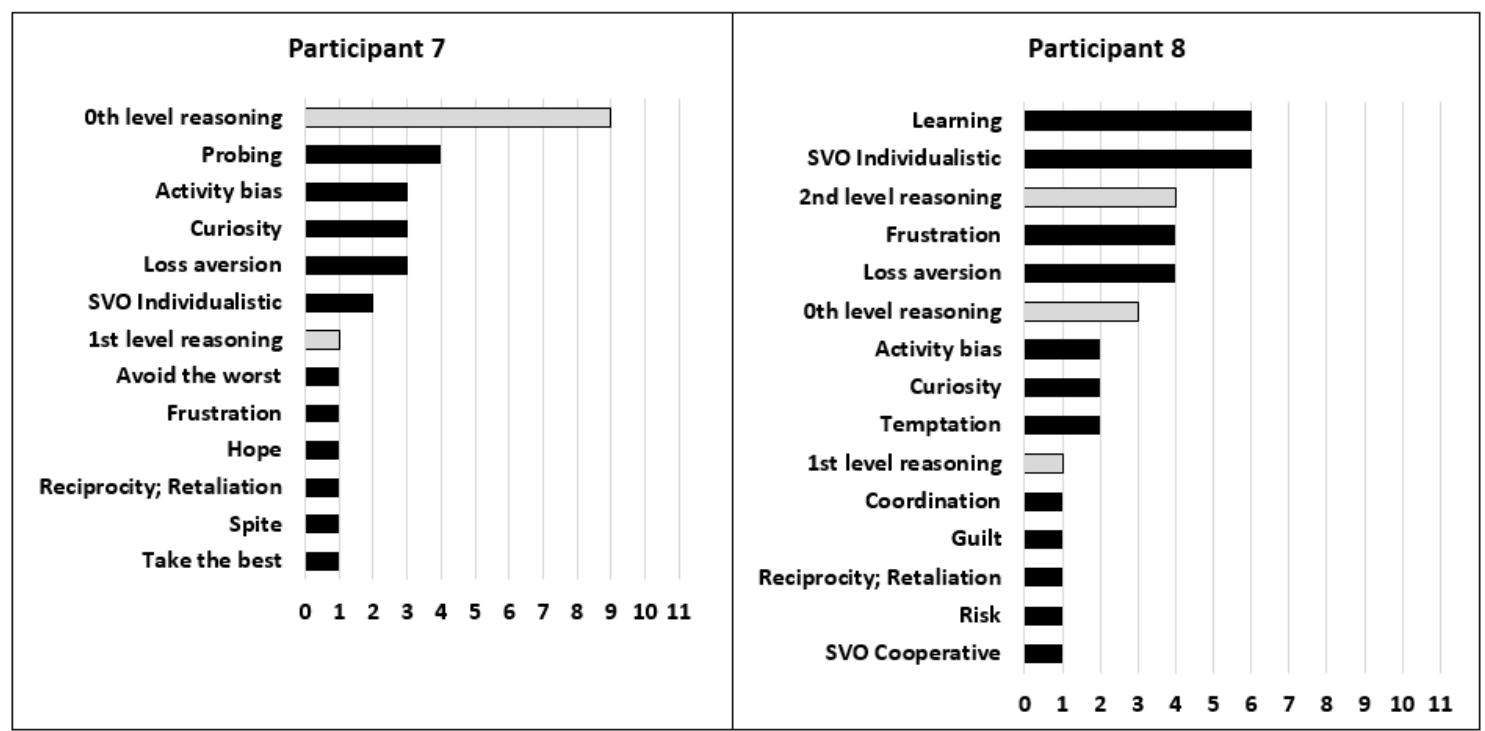

Figure 5. Number of coding references for Pair 4's levels of reasoning (grey bars) and motives (black bars).

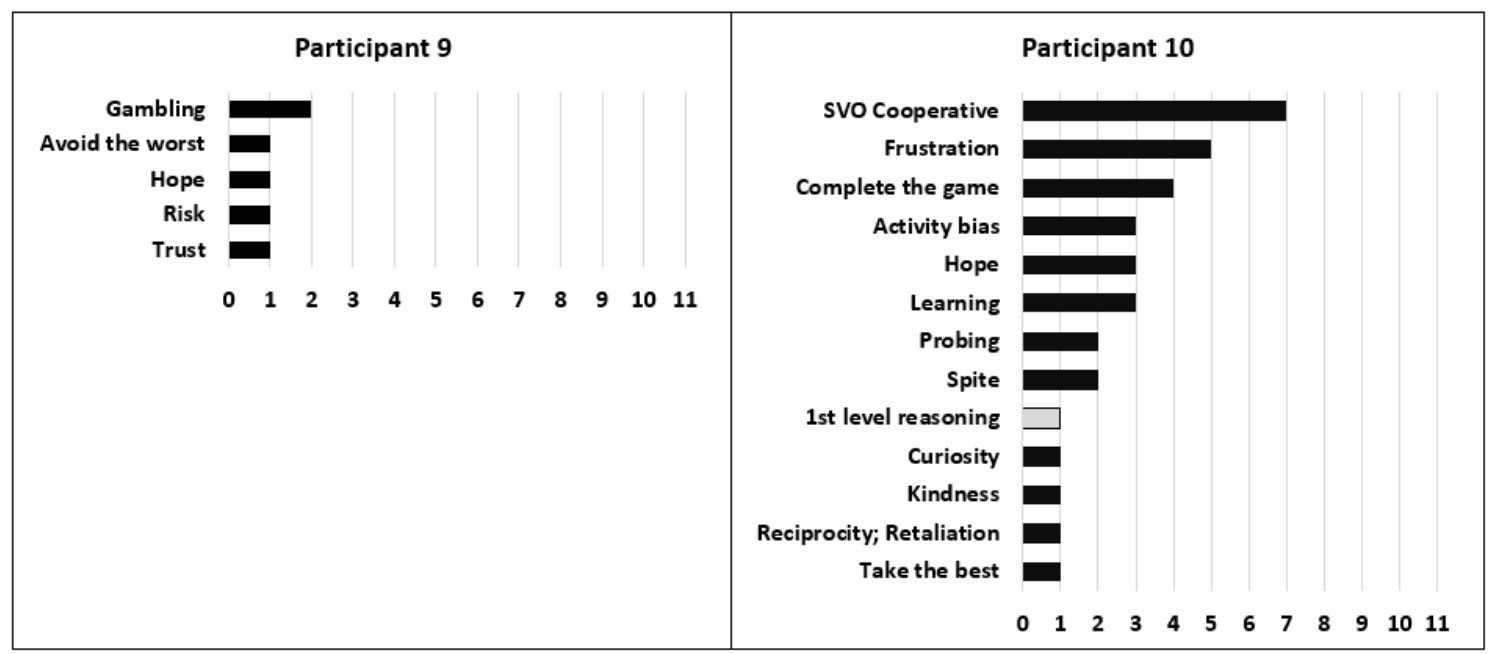

Figure 6. Number of coding references for Pair 5's levels of reasoning (grey bar) and motives (black bars).

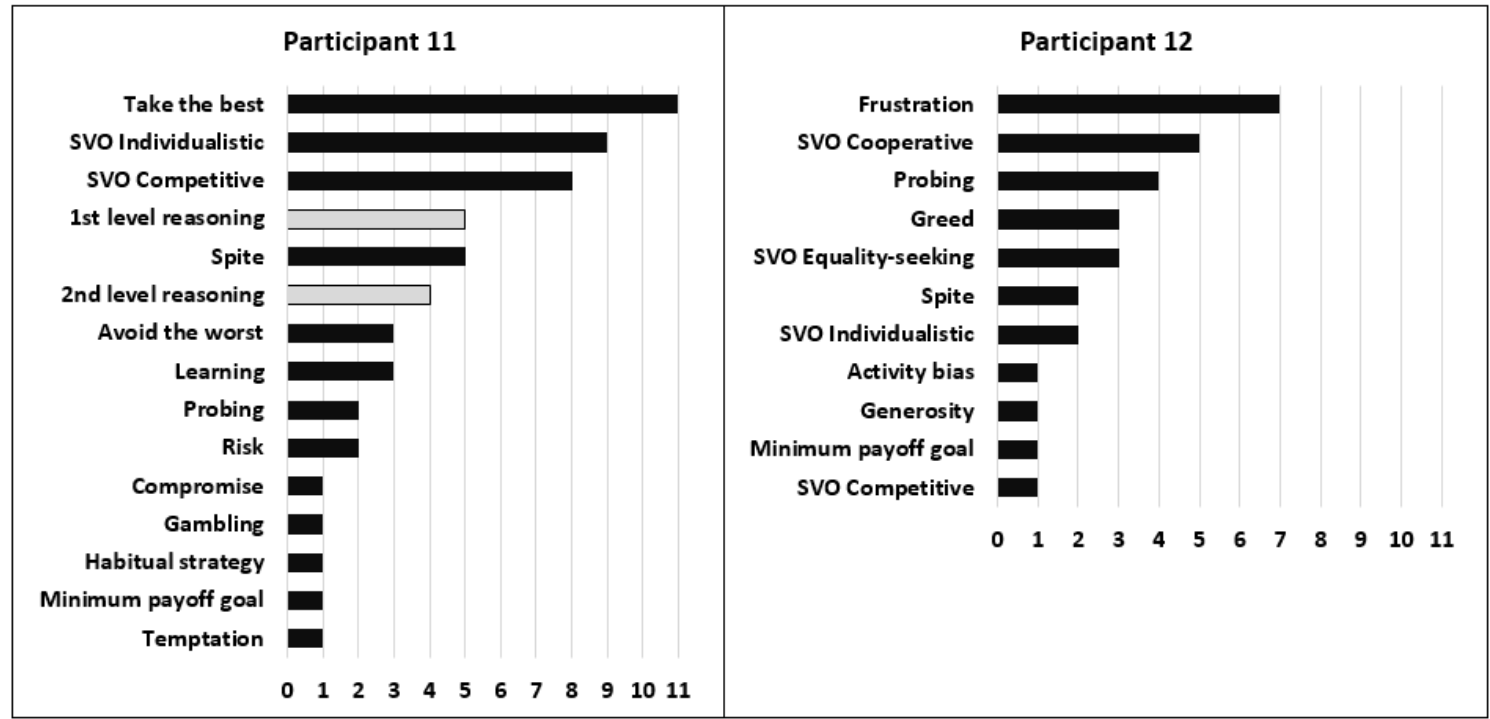

Figure 7. Number of coding references for Pair 6's levels of reasoning (grey bars) and motives (black bars). 
The verbalisations also differed in type of content, ranging from categories requiring lower levels of processing such as 'reading off screen' or 'descriptive talk' to more profound categories such as 'deliberation about the game' or 'reflection about other player'. In two cases (Participants 3 and 9), the majority of talk was purely descriptive. All other participants, however, spent the majority of their time engaging in analyses of the game or the co-player's behaviour.

In addition to the overall quantity and quality of the verbalisations, their contents were analysed against the background of three different themes: levels of reasoning, motivation, and study concerns. These are summarised below.

Levels of reasoning. The verbal records yielded evidence for all four possible levels of reasoning in the 6-node Centipede game (0th, 1st, 2nd, and full BI reasoning). Table 1 shows that the majority of participants used some iterated reasoning, with eight participants demonstrating a minimum of 1st-level reasoning on at least one occasion during the testing session. Two (Participants 8 and 11) showed some 2nd-level reasoning; Participant 11, for example, reasoned as follows:

So . . I might think about stopping at 1.60 , actually. Because if I were in the other room, I'd be thinking that the other person will be stopping at $6.40 \ldots$ umm . . But then again, there's 25.60 at the end . . . Soo ... I'll click GO.

Furthermore, one player (Participant 2) demonstrated full BI reasoning for the supergame consisting of the 10 repetitions of the game:

If I keep stopping at 6, and they're gonna. . . . Yeah, it's probably gonna escalate and they're gonna want to STOP then at 5 and yeah, then all the way back. It's probably better to go for 6.40 now, even though that's less than 12.80 [Player B's potential maximum payoff] because, um, at least that way, um, it should go quite smoothly perhaps. 'Cause if I try and STOP and get my 12.80, then they'll realise that's what I'm doing, and they'll get back to their 6.40 probably.

Interestingly, however, higher-level reasoning did not lead to earlier defection as predicted by classical game theory. As evident from the example, Participant 11, despite considering an exit move at Node 3 that would have rendered a personal payoff of $£ 1.60$, was too tempted by the $£ 25.60$ dangling from the Centipede's final leg to defect. Similarly, Participant 2 decided to go all the way to the game's natural end with a personal payoff of $£ 6.40$ rather than defecting at Node 6 , which would have yielded this player's maximum of $£ 12.80$, in order to prevent the game from unravelling.

Motives. Across the 12 participants, no fewer than 32 distinguishable motives for decisions were identified (see Table 1). This most important finding is the surprisingly large variety of different motives affecting play in the game. Five of the motives correspond to standard SVO categories. The individualistic SVO was by far the most common, with 33 coding references across 10 different participants. For example, Participant 3 in role of Player B said: 'Yes, and I STOP here. . . . Yeah, 'cause I won't ever do the last GO because, um ... I have no benefit from it. So ... I think I'm always gonna keep with this 6th decision.' The second most frequent SVO was the cooperative orientation with 24 coding references across seven participants. For example, Participant 5 said:

Do you know what, I'm just gonna, um, go for it, just carry on cause I figure out I do alright for many of these now. And he does all right for. . . . So I think he's . . ohh! . . . Do you know what, I'm gonna . .. I'm gonna go for GO and then we can both make some good money.

This was followed by the equality-seeking orientation with 19 coding references across five participants. For example, Participant 1 said: 'They've been so nice to me. Yeah . . . they've 
been so nice to be over the ... over whole the study, soo . . . It's only . . . it's only fair that they get some of the payoffs as well.'

Following in frequency was the competitive SVO with 13 coding references across three participants. For example, Participant 11 said:

So if I was them, I would probably STOP at 3.20 next because they know I wanna GO for 6.40 again. So now I'm just gonna try and mess with their heads a little bit. I'll STOP at 1.60. Which I know that gets me less potentially but it's still more than they would have got . . . of the total ... if that makes sense.

Least frequent was the altruistic SVO with seven coding references across three participants. Participant 5 said: 'And I'm gonna GO again. And ... I think he's gonna GO again . . . ah yeah .... And I'm gonna GO again as well. So, it's looking good for both of us. Obviously a bit better for him than for me.'

Importantly, however, not a single participant was characterised by one orientation only. Instead, a range of value orientations and additional motives informed choices in the game, with up to 16 different motives identified for a single participant (see Figures 2-7). These included (in descending order of coding frequency): 'Take the best' (going for the highest payoff possible in the game); 'Frustration'; 'Probing'; (testing the other player's behaviour) and 'Learning from previous experience'.

Motives and context. To investigate motives and specific key moves in the game ('GO'; 'GO at first node'; 'GO at last node'; 'STOP'; and 'STOP at first node'), the coding references for the motivations and the different moves were cross-tabulated with the help of a NVivo Matrix Coding Query (see Table 4).

The motive most frequently associated with a general GO move (at any decision node) was the cooperative SVO (14 coding references). This was closely followed by 'Take the best' ( 11 coding references) and 'Probing' (10 coding references). The latter refers to the aim of assessing the co-player's behaviour and predicting future moves on that basis. Participant 10, for example, said:

Now they've chosen to GO as well. Umm, I'm gonna keep going. I like to just see in this one how long I can keep the other person going for it as well. . . . The decision they'll make in this one will be interesting to see what I think they're gonna do for the next ten.

GO moves at the first decision node are of particular theoretical importance, because orthodox game theory predicts an immediate STOP move at Node 1. In our data, the most commonly motive linked to an initial GO move was activity bias. For example, Participant 10 said: 'OK, OK . . . so the first one is blue so it's my turn. I choose to GO or STOP. Umm I keep going, umm, 'cause this is the first one, so I might as well GO and see what happens.'

The most frequent motive associated with a GO move at the last decision node leading to the natural end of the game was the cooperative SVO. Additional motives associated with cooperation at the last decision node were the altruistic SVO and a wish to complete the game. Participant 10 said: 'Umm, I'm gonna keep going. My aim is to try and get all the way to the end just to feel like I've finished it but I think the other person is gonna keep stopping at what benefits them.'

A general STOP move (at any decision node) was most frequently associated with an individualistic SVO. For example, Participant 8 commented: 'I'm gonna STOP here, umm, so that if this round is chosen to pay me, then I'll probably be paid, umm, a good amount.' Another coding category commonly linked with STOP was the learning from previous experience in the game; For example, Participant 8 said: 
Oh, so he's decided to oh ... 'The decision sequence has been ended' . . . so he decided to STOP now. Ugh, maybe I should have just stopped there. But it's fine, like, umm, I've actually lost 40p but it's, it's OK. I can just be careful next time.

A STOP move at the first decision node occurred only once across 60 games, and was linked to frustration, retaliation, and spite on the part of both players. Participant 7 explained the immediate exit move as follows: 'So because they keep pressing STOP, I'm going to press STOP on the first go'; and Participant 8's reaction was: 'Oh he stopped it right at the first go! [Laughter] I'm getting angry now!'

Motivation and reasoning level. With the help of another Matrix Coding Query, motives were cross-tabulated with reasoning levels to investigate shared coding references and thus explore the relationship between levels of reasoning and motives (see Table 5). A strong association between 0th-level reasoning and the following motives became evident: 'activity bias', 'curiosity', 'probing', and 'loss aversion', indicating that low-level reasoners typically used loss-sensitive trial-and-error strategies. For example, Participant 3 said: 'I decide to GO ... a and the Participant A chose to GO . . . and ugh OK. I think I'm going to STOP. ... So that there's more action [laughter].' Participant 7 said: 'And again, if they click GO, then I will click GO again to see what they do on the next sequence .. . 'cause I'm a bit curious like that.'

Study concerns. The last coding category is a collection of verbalisations regarding various aspects of the research design and task variables that could prove useful in refining existing experimental methods and procedures. Example statements are provided in Table 6.

Most participants showed excellent understanding of the Centipede game's rules and payoff structure following the detailed task instructions, even though some participants seemed to expect a change in player roles from one game to another in spite of having been instructed otherwise. The majority of participants appeared to have accepted the task instructions received by the experimenter. Only two participants, whose co-players behaved in ways quite contrary to their expectations, began to challenge the information that they had been given.

Interestingly, eight participants (with a total of 24 individual coding references) interpreted the co-player's response latency as an important signal about the co-player's intentions. Participant 5, for example, commented:

Ooh, he's taking a bit longer to decide this time . . . umm . . . interesting. . . . Not sure why he's delaying it. Was a bit worried there, wondering what he was up to. . . But he seems to have gotten back on to track.'

Participant 12 even manipulated his own response times to confuse his co-player: 'Let's take five seconds to think. Actually, I'm not going to think. I'm just going to wait here for five seconds to make them think that I'm thinking. And then ... see what happens.

\section{Discussion}

Cooperation levels in the present study were marginally higher than levels reported in previous studies using the same version of the Centipede game. The quantitative results show a mean exit node of 5.12, as compared to 4.27 (McKelvey \&Palfrey, 1992) and 4.00 (Kawagoe \& Takizawa, 2012). This could be explained by our use of fixed participant pairing for all ten rounds of the game rather than random re-matching after each round. In a direct comparison of fixed pairing versus random re-pairing in Centipede games, Pulford, Colman, Lawrence, and Krockow (2015) found higher cooperativeness in the fixed-pairing condition, and this was explained in terms of direct reciprocity and trust-building across rounds.

The results of the verbal protocol analysis provide strong support for the existence of different levels of rationality as well as different types of SVOs among the participants. In 
accordance with previous research (e.g., Nagel, 1995), the majority of participants used 1stlevel reasoning when trying to derive an optimal strategy. Furthermore, there was evidence for five different types of SVO motivating the participants' moves, with individualistic concerns being the most common, followed by cooperative, equality-seeking, competitive, and altruistic orientations. These findings are in line with previous results by Au and Kwong (2004), who assessed the occurrence of the three main SVOs across different countries and found that $57 \%$ of people were motivated predominantly by prosocial concerns (including cooperative, equality-seeking and altruistic), $27 \%$ by individualistic, and $16 \%$ by competitive orientations.

However, despite the qualitative support for the theoretical concepts, the verbal records showed that almost all participants engaged in multiple levels of reasoning and were influenced by several different SVOs, depending on the situation and their previous experience in the game. Furthermore, the data provided evidence for a wealth of additional motives for choice that mingled and interacted to determine an individual's moves. To illustrate the variety of factors and their complex interplay when making decisions in the Centipede game, the comparison of the verbal records generated by two player pairs with ostensibly similar strategies (as shown by almost identical game outcomes) is instructive.

Comparison of Pairs 1 and 3 . The quantitative results for the games completed by

Pair 1 and Pair 3 were very similar. Both pairs demonstrated high levels of cooperation across rounds (apart from Rounds 9 and 10 completed by Pair 1), generally reaching the game's natural end. Given these similar quantitative results, one might expect similar motives or reasoning levels to have influenced the respective players' choices. In fact, the pairs were radically different. The players of Pair 1 were among the most sophisticated decision makers in our study. Both used iterated reasoning to choose moves in the game, and Player B (Participant 2) was the only participant to have demonstrated perfect BI reasoning in the study:

So yeah, I'm basically halving what I can get at the end because I feel that if I stopped at 6 , they'd realise that's what I was doing and then perhaps STOP at 5 and then . . . and you know . . . and I guess the way this is set up means that it would then, you know, cascade backwards almost.

Despite successfully identifying the subgame perfect Nash equilibrium, Player B chose to cooperate and compromise, even choosing an altruistic GO move at the final decision node, in an attempt to prevent the game unravelling and yielding a minimal BI payoff. Player B further considered a premature STOP move by Player A unlikely due to the game's negligible payoffs at early exit nodes:

See, I don't think there's gonna be any decision making here, really, 'cause we want to GO. It's gonna be at 4 or 5 that the decision will be made, really. I don't know why they would want to STOP at 3 .

Only on Rounds 9 and 10 did Player B decide to maximise his personal payoff and STOP at the last decision node, because the opportunities for Player A to retaliate were minimal at this point. Player B explained: 'So I think I'll STOP at 6 on the 10th go. So then I'll have a 10\% chance of getting the 12.80 but there's no point of doing it before that because they might STOP at 5.'

Pair 1's Player A initially chose to cooperate out of a recognition of the need for teamwork in order to get to the high payoffs towards the end of the game, all the while hoping the other person would play along:

Um, kind of tempted to carry on . . kind of tempted to carry on because I'll get half and they'll get a massive bonus. So I'll see . . I'll be nice to them and we'll see what happens. We'll see how they react. 
As Player B kept cooperating at the final decision node, Player A was struck by his kind nature, expressed a high level of gratitude, and increasingly reported unease at the accruing payoff inequality between the two players. Consequently, Player A appeared almost relieved that Player B finally opted to counteract the payoff imbalance and stopped earlier for rounds 9 and 10: 'Ahhh yes, "chose to STOP”. There you go! I do not blame you. I don't blame you! I have been ... I have been . . . It's only fair!'

Looking at the verbalisations by Pair 3 of this study, neither player demonstrated any form of iterated reasoning. Player A (Participant 6) was particularly myopic in choosing a strategy in the game:

OK ... sooo. I'm gonna press GO again. I don't know a reason why not to again. If the other participant said he can continue then I might as well choose GO. OK, I'm waiting. . . He said GO again. So, umm, I'm gonna continue, too.

Player B (Participant 5), instead of engaging in recursive reasoning on the other player's likely aims and choices, merely hoped for reciprocal cooperation:

I can see the only way that I'm gonna make . . umm . . the best way for me to make some money is to get all the way to the end umm the further up I can go umm. . . So I'm gonna GO on this one I think and hope for the best.

The decision to cooperate at the final decision node was driven by a highly prosocial (cooperative and altruistic) SVO and the wish to complete the game. Furthermore, after the first round of the game, Player B continued with his initial strategy and made all following decisions comparatively quickly and with little additional deliberation.

Player A, on the other hand, initially chose to cooperate based on mere curiosity. Following a few rounds of the game, however, she became increasingly perturbed by the identical payoffs and her repeated personal gains, consequently starting to question the set-up of the study: 'Umm. I'm really sorry to have second thoughts about this. . . . I don't know. . . . It doesn't seem right for me to be winning all the time. Winning in the sense of making more money than him.' The individualistically orientated Player A continued to choose GO because it seemed to be rewarding, but was deeply perplexed about Player B's reason for continually choosing GO.

This detailed comparison of two player pairs with very similar quantitative results reveals the variety of cognitive processes and considerations underlying any one strategy, powerfully showcasing how identical moves can be interpreted in drastically different ways. This qualitative study showed that no one move was associated with a particular level of rationality or a motive. There was no evidence that sophisticated recursive reasoning skills led to the equilibrium outcome of immediate defection in the Centipede game. In fact, those participants with higher-level reasoning skills - and in particular the one participant with full BI reasoning - cooperated more consistently than subjects of lesser strategic depth who frequently opted for haphazard trial-and-error strategies. These qualitative results therefore call into question previous empirical findings on backward induction play by expert Chess players with supposedly advanced levels of reasoning (Palacios-Huerta \& Volij, 2009). Furthermore, despite lending support to the existence of different types of rationality as assumed by ToM, the findings challenge these types' predictive power in the context of the Centipede game.

When examining the effects of different motives, a closer look at the context of the move is necessary to detect patterns in the verbal data. A mere activity bias appeared to be the most frequent motive for cooperation at the first decision node. Many participants considered the initial GO move an obvious, almost non-debatable choice. Furthermore, early 
GO moves were generally linked to a general pursuit of high numbers, because most participants considered the payoffs at the first few terminal nodes insubstantial. This finding lends support to previous research demonstrating the importance of stake size in Centipede games (Rapoport, Stein, Parco, \& Nicholas, 2003). Motives such as prosocial value orientations usually became important only towards the end of the game and, alongside a wish to complete the game, seemed to have a particularly strong influence on the decision at the final decision node.

Similarly, whereas there were many motives for general STOP moves in the game, with a majority related to individualistic concerns about personal risks and losses, there was a strong link between immediate defection at Node 1 and spite, frustration, and retaliation. The game-theoretic outcome was therefore interpreted as a particularly competitive move.

With regard to the research design and materials, the verbalisations demonstrated that the instructions on the game's rules were well understood and generally believed by all participants. However, details (e.g., regarding the assignment of player roles) were occasionally overlooked and could be made more prominent in future instruction slides.

An important aspect of the design — shared with most published Centipede experiments - was the flexibility of permitted response times that allowed for indefinite delays, potentially carrying non-verbal timing signals. Our qualitative data revealed that human decision makers attach meaning to pauses made by their co-players and that they also sometimes deliberately use delays as covert signals of their own intentions or emotions, such as discontent. Future studies should therefore control such means of communication and impose standardised response times in order to circumvent covert signalling between participants.

Overall, this study demonstrates the potential merits of qualitative analyses for behavioural game theory. All participants produced intelligible verbalisations that provided powerful evidence for a complex interplay of large numbers of different reasoning levels and motives. The range of motives was much larger than could ever be accounted for by econometric models, and the qualitative data provide evidence for previously neglected motives, such as activity bias. Our findings also point to the need for design improvements, such as the control of permitted response latencies. Future research should apply qualitative methods to a wider range of experimental games, with a focus on other mixed-motive games in which motives for choices are likely to be complex.

\section{References}

Au, W. T., \& Kwong, J. Y. Y. (2004). Measurements and effects of social-value orientation in social dilemmas: A review. In R. Suleiman, D. Budescu, I. Fischer. \& D. Messick (Eds.), Contemporary Psychological Research on Social Dilemmas (pp. 71-98). Cambridge, UK: Cambridge University Press.

Aumann, R. J. (1995). Backward induction and common knowledge of rationality. Games and Economic Behavior, 8(1), 6-19. doi:10.1016/S0899-8256(05)80015-6

Aumann, R. J. (1998). On the Centipede game. Games and Economic Behavior, 23(1), 97-105. doi:10.1006/game.1997.0605

Balliet, D., Parks, C., \& Joireman, J. (2009). Social value orientation and cooperation in social dilemmas: A meta-analysis. Group Processes Intergroup Relations, 12, 533-547. doi:10.1177/1368430209105040

Bornstein, G., Kugler, T., \& Ziegelmeyer, A. (2004). Individual and group decisions in the Centipede game: Are groups more "rational" players? Journal of Experimental Social Psychology, 40, 599-605. doi:10.1016/j.jesp.2003.11.003

Camerer, C. F., Ho, T., \& Chong, J.-K. (2004). A cognitive hierarchy model of games. Quarterly Journal of Economics, 119, 861-898. doi:10.1162/0033553041502225

Colman, A. M., Pulford, B. D., \& Lawrence, C. L. (2014). Explaining strategic coordination: Cognitive hierarchy theory, strong Stackelberg reasoning, and team reasoning. Decision, 1, 35-58. doi: $10.1037 / \mathrm{dec} 0000001$ 
Doshi, P., Qu, X., Goodie, A. S., \& Young, D. L. (2012). Modeling human recursive reasoning using empirically informed interactive partially observable Markov decision processes. IEEE Transactions on Systems, Man, and Cybernetics - Part A: Systems and Humans, 42(6), 1529-1542. doi:10.1109/TSMCA.2012.2199484

Droste, E., Kosfeld, M., \& Voorneveld, M. (2003). Best-reply matching in games. Mathematical Social Sciences, 46, 291-309. doi:10.1016/S0165-4896(03)00065-9

Ericsson, K. A, \& Simon, H. A. (1993). Protocol analysis: Verbal reports as data. Cambridge, MA: MIT Press.

Ericsson, K. A, \& Simon, H. A. (1998). How to study thinking in everyday life: Contrasting think-aloud protocols with descriptions and explanations of thinking. Mind, Culture, and Activity, 5, 178-186. doi: 10.1207/s15327884mca0503_3

Goodie, A. S., Doshi, P., \& Young, D. L. (2012). Levels of theory-of-mind reasoning in competitive games. Journal of Behavioral Decision Making, 25, 95-108. doi:10.1002/bdm

Hedden, T., \& Zhang, J. (2002). What do you think I think you think? Strategic reasoning in matrix games. Cognition, 85, 1-36.

Ho, T.-H., \& Su, X. (2011). A dynamic level-k model in Centipede games. Management Science, 59, 452-469. doi:abs/10.1287/mnsc.1120.1645

Kawagoe, T., \& Takizawa, H. (2012). Level-k analysis of experimental Centipede games. Journal of Economic Behavior \& Organization, 82, 548-566. doi:10.1016/j.jebo.2012.03.010

Kelley, H. H., \& Thibaut, J. W. (1978). Interpersonal relations: A theory of interdependence. New York: Wiley.

Levitt, S. D., List, J. A., \& Sadoff, S. E. (2011). Checkmate: Exploring backward induction among chess players. American Economic Review, 101, 975-990. doi:10.1257/aer.101.2.975

McClintock, C. G. (1972). Social motivation: A set of propositions. Behavioral Science, 17, 438-454. doi:10.1002/bs.3830170505

Messick, D. M., \& McClintock, C. G. (1968). Motivational bases of choice in experimental games. Journal of Experimental Social Psychology, 4, 1-25. doi: 10.2307/2951567

Nagel, R. (1995). Unraveling in guessing games: An experimental study. The American Economic Review, 85, 1313-1326.

Nagel, R., \& Tang, F. (1998). Experimental results on the Centipede game in normal form: An investigation on learning. Journal of Mathematical Psychology, 42, 356-384. doi:10.1006/jmps.1998.1225

NVivo Qualitative Data Analysis Software (Version 10) (2012). Melbourne, Australia: QSR International.

Palacios-Huerta, I., \& Volij, O. (2009). Field Centipedes. American Economic Review, 99, 1619-1635. doi:10.1257/aer.99.4.1619

Pulford, B. D., Colman, A. M., Lawrence, C. L., \& Krockow, E. M., (2015). Reasons for cooperating in repeated interactions: Social value orientations, fuzzy traces, reciprocity, and activity bias. Unpublished manuscript, School of Psychology, University of Leicester, UK.

Pulford, B. D., Krockow, E. M., Colman, A. M., \& Lawrence, C. L. (2015). Social value induction and cooperation in the Centipede game. Unpublished manuscript, School of Psychology, University of Leicester, UK.

Rapoport, A., Stein, W. E., Parco, J. E., \& Nicholas, T. E. (2003). Equilibrium play and adaptive learning in a three-person Centipede game. Games and Economic Behavior, 4, 239-265. doi:10.1016/S08998256(03)00009-5

Rosenthal, R. W. (1981). Games of perfect information, predatory pricing and chain store paradox. Journal of Economic Theory, 25, 92-100. doi:10.1016/0022-0531(81)90018-1

Stahl, D. O., \& Wilson, P. W. (1995). On players' models of other players: Theory and experimental evidence. Games and Economic Behavior, 10, 218-254. doi:10.1006/game.1995.1031

Van Lange, P. A. M. (1999). The pursuit of joint outcomes and equality in outcomes: An integrative model of social value orientation. Journal of Personality and Social Psychology, 77, 337-349. doi:10.1037//0022-3514.77.2.337

Zhang, J., Hedden, T., \& Chia, A. (2012). Perspective-taking and depth of theory-of-mind reasoning in sequential-move games. Cognitive Science, 36, 560-73. doi:10.1111/j.1551-6709.2012.01238.x 
Table 1. Hierarchical coding node structure for 'Levels of reasoning' and 'Motives' including example statements, number of coding sources, and number of individual coding references

\section{Number of sources Number of \\ (participants) coding}

references

\section{Levels of reasoning}

Oth level

P6: 'OK. . sooo. I'm gonna press GO again. I don't know a reason why not to again. If the other participant said he can continue then I might as well choose GO.'

1st level

P3: 'OK, and now I'll STOP at the fourth decision because Participant A . . . he's more likely to STOP a the fifth decision . . so . . yeah, I'll STOP at the fourth decision.'

2nd level

P11: 'So . . I might think about stopping at 1.60 actually. Because if I were in the other room, I'd be thinking that the other person will be stopping at 6.40.'

BI reasoning

P2: 'If I keep stopping at 6, and they're gonna . . Yeah, it's probably gonna escalate and they're gonna want to STOP then at 5 and yeah, then all the way back.'

\section{Motives}

SVO Individualistic

P3: 'Yes, and I STOP here. . . Yeah, 'cause I won't ever do the last GO because, um ... I have no benefit from it. So . . I think I'm always gonna keep with this 6th decision.'

SVO Cooperative

P5: 'I'm gonna go for GO and then we can both make some good money.'

SVO Equality-seeking

P1: 'They've been so nice to me. Yeah . . they've been so nice to be over the ... over whole the study, soo. . . It's only . . it's only fair that they get some of the payoffs as well.'

Take the best

P1: 'Yeah, they pressed GO and obviously I press GO as well cause I want the maximum pay-out which is at the end.'

Frustration

P10: 'Seventh one and I'm still Participant A. They still keep stopping. Arghh!'

Probing

P10: 'I like to just see in this one how long I can keep the other person going for it as well. . . The decision they'll make in this one will be interesting to see what I think they're gonna do for the next ten.'

Learning

P8: 'Umm so he's gone and now it's my turn. Um ohh it's a tricky one. I think last time I let him go and then he stopped so I'm going to STOP this one here now.'

Habitual strategy

P5: 'Oh, OK, it's the same sequence as last time. So it's about getting as far ... as far as I can. Hopefully . . it looks like he might do the same thing. We just try to get to the end.' Spite

P11: 'Although [Laughter], were it me, and I knew that the person in the other room was basically stopping me from getting quite a lot, I'd be tempted to just... . mess it up for them and click a really low value [Laughter]... .' 
SVO Competitive

3

P11: 'So now I'm just gonna try and mess with their heads a little bit. I'll

STOP at 1.60. Which I know that gets me less potentially but it's still more than they would have got... of the total if that makes sense.'

Reciprocity/retaliation

P8: 'So it's my turn. Oh I have to STOP it here because I had a few GOs

and he does it all the time so... ok.'

Activity bias

P4: 'I want to see if we can take it to the end. Purely for fun, I think.

Regardless of the money that's at the bottom.'

Avoid the worst

P2: 'And I don't think they'd be happy with 3.20 really when 25.60 is just around the corner.'

Kindness

P1: 'If I do the kind thing and GO on then that person will STOP. They will completely ignore my kind deed of not stopping here whereas I will get a much, much higher proportion of. . .'

Hope

P10: 'I'm hoping if I keep trying . . yeah ... I'm hoping that if I keep trying then they won't. Ahh yeah, they keep stopping'

Loss aversion

P6: 'Really tempted to press STOP to see what would change umm but that would mean that I would probably lose and I'm not sure I wanna lose, yet.'

Curiosity

P7: 'And again, if they click $G O$, then I will click $G O$ again to see what they do on the next sequence . . cause I'm a bit curious like that.'

SVO Altruistic

P5: 'And I'm gonna GO again as well. So, it's looking good for both of us. Obviously a bit better for him than for me.'

Risk

P3: 'And ugh, I think I'm going to STOP again because the difference here is, um, higher and this is, um, at the end. This is a more, um, risk to take so I'm going to STOP at this 6th decision.'

Temptation

P6: 'Really tempted to press STOP to see what would change umm but that would mean that I would probably lose and I'm not sure I wanna lose, yet. '

Completing the game

P10: 'Umm I'm gonna keep going. My aim is to try and get all the way to the end just to feel like I've finished it.'

Generosity

P2: 'Well, if they STOP at 5 now, that's sort of like a rude move really cause I've been letting them get 25.60 all those times but we'll see how generous they're feeling really.'

Gambling

P4: 'Actually, I wanna do third time lucky. I wanna see if this person will take, will GO to the end third time around. And if they don't, I'm just gonna STOP it at number 5 next time. . . It's like gambling, this!' 
Minimum payoff goal 4

P11: 'So there's a potential that they're gonna STOP at 4. At 3.20 and that would leave me with 80 p but I don't think they will. I don't think 3.20 is enough.'

Compromise

P4: 'I think if the next person clicks GO, I will click GO at 3 because financially, I'd come slightly better off. Although not as better off as if we went to question 5.'

Guilt

P4: 'So now I have to be generous, I feel. Click GO again. And then ... I would feel bad if I stopped at 3 again.'

Greed

P12: 'So they STOP at 5. Oh, all right! They probably think I'm greedy as well! Oh yeah, oh, alright, OK.'

Coordination

P1: 'Well to me this looks like a . . well, not like a puzzle but like a task that requires unspoken coordination from both the players for maximum payoff.'

Trust

P9: 'I can chose to GO or STOP', um, I'm going to GO . . Hopefully . . . come on . . yes! Ummm . . . How much do I trust A? . . Come on, come on.' 
Table 2. Exit nodes across ten rounds (R1-R10) of the Centipede game for six pairs of participants

\begin{tabular}{|c|c|c|c|c|c|c|c|c|c|c|c|c|}
\hline & & R1 & R2 & R3 & $\mathrm{R} 4$ & R5 & R6 & R7 & R8 & R9 & R10 & $\begin{array}{l}\text { Number of } \\
\text { STOP } \\
\text { moves }\end{array}$ \\
\hline Pair 1 & $\begin{array}{l}\text { Participant 01; A } \\
\text { Participant 02; B }\end{array}$ & 7 & 7 & 7 & 7 & 7 & 7 & 7 & 7 & 6 & 6 & $\begin{array}{l}0 \\
2\end{array}$ \\
\hline Pair 2 & $\begin{array}{l}\text { Participant 03; B } \\
\text { Participant 04; A }\end{array}$ & 3 & 4 & 5 & 6 & 6 & 6 & 5 & 5 & 4 & 3 & $\begin{array}{l}5 \\
5\end{array}$ \\
\hline Pair 3 & $\begin{array}{l}\text { Participant 05; B } \\
\text { Participant 06; A }\end{array}$ & 7 & 7 & 7 & 7 & 7 & 7 & 7 & 7 & 7 & 7 & $\begin{array}{l}0 \\
0\end{array}$ \\
\hline Pair 4 & $\begin{array}{l}\text { Participant 07; A } \\
\text { Participant 08; B }\end{array}$ & 4 & 3 & 2 & 3 & 2 & 2 & 2 & 1 & 2 & 2 & $\begin{array}{l}3 \\
7\end{array}$ \\
\hline Pair 5 & $\begin{array}{l}\text { Participant 09; B } \\
\text { Participant 10; A }\end{array}$ & 6 & 4 & 5 & 6 & 6 & 2 & 4 & 6 & 6 & 6 & $\begin{array}{l}9 \\
1\end{array}$ \\
\hline Pair 6 & $\begin{array}{l}\text { Participant 11; A } \\
\text { Participant } 12 ; \mathrm{B}\end{array}$ & 5 & 3 & 5 & 5 & 5 & 5 & 5 & 5 & 5 & 5 & $\begin{array}{c}10 \\
0\end{array}$ \\
\hline
\end{tabular}


Table 3. Number of coding references per reasoning level and motive for each participant.

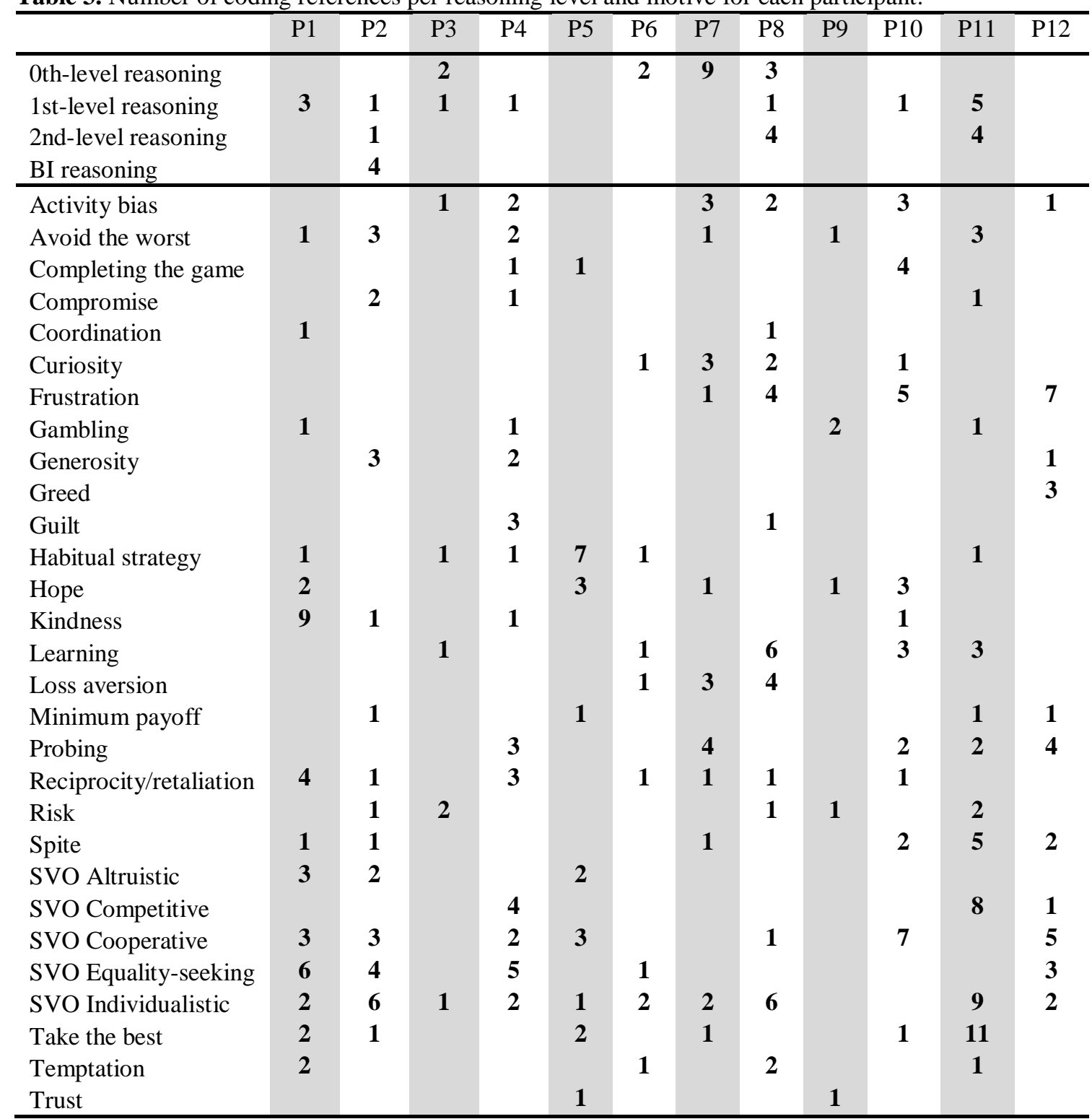


Table 4. Results of NVivo matrix coding query: Frequencies of coding references of motives across five specific decision nodes in the game

\begin{tabular}{|c|c|c|c|c|c|}
\hline & $\mathrm{GO}$ & $\begin{array}{c}\text { GO at } \\
\text { first node }\end{array}$ & $\begin{array}{c}\text { GO at } \\
\text { last node }\end{array}$ & STOP & $\begin{array}{l}\text { STOP at } \\
\text { first node }\end{array}$ \\
\hline Activity bias & 4 & 6 & 1 & 1 & 0 \\
\hline Avoid the worst & 3 & 1 & 0 & 3 & 0 \\
\hline Completing the game & 4 & 0 & 4 & 1 & 0 \\
\hline Compromise & 1 & 0 & 0 & 2 & 0 \\
\hline Coordination & 1 & 0 & 0 & 0 & 0 \\
\hline Curiosity & 3 & 2 & 0 & 1 & 0 \\
\hline Frustration & 8 & 0 & 1 & 9 & 2 \\
\hline Gambling & 3 & 0 & 0 & 2 & 0 \\
\hline Generosity & 4 & 0 & 1 & 1 & 0 \\
\hline Greed & 1 & 0 & 0 & 3 & 0 \\
\hline Guilt & 2 & 0 & 0 & 2 & 0 \\
\hline Habitual strategy & 7 & 0 & 5 & 0 & 0 \\
\hline Hope & 6 & 2 & 2 & 1 & 0 \\
\hline Kindness & 4 & 0 & 3 & 4 & 0 \\
\hline Learning & 3 & 0 & 1 & 10 & 0 \\
\hline Loss aversion & 1 & 0 & 0 & 7 & 0 \\
\hline Minimum payoff & 2 & 0 & 1 & 0 & 0 \\
\hline Probing & 10 & 1 & 0 & 2 & 0 \\
\hline Reciprocity/retaliation & 1 & 0 & 0 & 5 & 1 \\
\hline Risk & 5 & 0 & 0 & 2 & 0 \\
\hline Spite & 1 & 0 & 0 & 8 & 1 \\
\hline SVO Altruistic & 3 & 0 & 4 & 0 & 0 \\
\hline SVO Competitive & 0 & 0 & 0 & 8 & 1 \\
\hline SVO Cooperative & 14 & 2 & 7 & 0 & 0 \\
\hline SVO Equality-seeking & 6 & 0 & 1 & 5 & 0 \\
\hline SVO Individualistic & 8 & 0 & 2 & 21 & 0 \\
\hline Take the best & 11 & 1 & 2 & 7 & 0 \\
\hline Temptation & 2 & 0 & 0 & 6 & 0 \\
\hline Trust & 2 & 0 & 1 & 0 & 0 \\
\hline
\end{tabular}


Table 5. Results of NVivo matrix coding query: Frequencies of coding references of motives across four levels of reasoning in the game

\begin{tabular}{lcccc}
\hline & Oth-level & 1st-level & 2nd-level & BI reasoning \\
\hline Activity bias & 5 & 0 & 0 & 0 \\
Avoid the worst & 1 & 1 & 0 & 1 \\
Completing the game & 0 & 0 & 0 & 0 \\
Compromise & 0 & 1 & 0 & 1 \\
Coordination & 0 & 0 & 0 & 0 \\
Curiosity & 5 & 0 & 0 & 0 \\
Frustration & 1 & 0 & 0 & 0 \\
Gambling & 0 & 0 & 1 & 0 \\
Generosity & 0 & 0 & 0 & 0 \\
Greed & 0 & 0 & 0 & 0 \\
Guilt & 0 & 0 & 1 & 0 \\
Habitual strategy & 0 & 0 & 0 & 0 \\
Hope & 0 & 0 & 0 & 0 \\
Kindness & 0 & 1 & 0 & 0 \\
Learning & 1 & 2 & 1 & 0 \\
Loss aversion & 4 & 1 & 0 & 0 \\
Minimum payoff & 0 & 0 & 0 & 0 \\
Probing & 4 & 1 & 1 & 0 \\
Reciprocity/retaliation & 1 & 0 & 0 & 0 \\
Risk & 0 & 1 & 0 & 0 \\
Spite & 0 & 1 & 2 & 0 \\
SVO Altruistic & 0 & 0 & 0 & 1 \\
SVO Competitive & 0 & 2 & 1 & 0 \\
SVO Cooperative & 0 & 0 & 0 & 0 \\
SVO Equality-seeking & 0 & 0 & 0 & 0 \\
SVO Individualistic & 2 & 6 & 3 & 0 \\
Take the best & 1 & 4 & 1 & 0 \\
Temptation & 0 & 2 & 0 & 0 \\
Trust & 0 & 0 & 0 & 0 \\
\hline & & & & 0 \\
\hline
\end{tabular}


Table 6. Hierarchical coding node structure for 'Study concerns' including example statements, number of coding sources, and number of individual coding references

\begin{tabular}{|c|c|c|}
\hline Study concerns & $\begin{array}{l}\text { Number of } \\
\text { sources } \\
\text { (participants) }\end{array}$ & $\begin{array}{l}\text { Number of } \\
\text { coding } \\
\text { references }\end{array}$ \\
\hline Timing clues & 8 & 24 \\
\hline $\begin{array}{l}\text { P5: 'Ooh he's taking a bit longer to decide this time ... umm ... interesting. } \\
\ldots \text { Not sure why he's delaying it. Was a bit worried there, wondering what he } \\
\text { was up to . . But he seems to have gotten back on to track.' }\end{array}$ & & \\
\hline $\begin{array}{l}\text { Misunderstanding instructions } \\
P 2: \text { 'So the reason I did that was because I imagine that these scores are } \\
\text { gonna switch over and I'll be able to win the } 25.60 \text { at some point and } \\
\text { perhaps if I let them win } 225 \text { now, in the future they'll let me GO.' }\end{array}$ & 5 & 10 \\
\hline $\begin{array}{l}\text { Comparing player roles } \\
\text { P11: 'And it's actually quite useful being Participant A because I get to make } \\
\text { the first decision, which kind of gives me a bit more power because I could } \\
\text { just STOP it now and still have more money than they do.' }\end{array}$ & 3 & 3 \\
\hline $\begin{array}{l}\text { Doubts about co-player being real person } \\
\text { P12: 'What? Did you see, this is starting to make me think that the other } \\
\text { person is not real. Who takes five... um five seconds to decide if they want } \\
40 p \text { ? Instead of maybe, don't know, } 1.60 \text { ?' }\end{array}$ & 1 & 3 \\
\hline $\begin{array}{l}\text { Doubts about payoff information } \\
\text { P6: 'So, anyway, still going. He's still going, too. So in order for him to still } \\
\text { be going and knowing that he's being paid less money, then that means that } \\
\text { he's probably seeing the same thing like me or something . . I I don't know. } \\
\text { Wouldn't make any sense.' }\end{array}$ & 1 & 3 \\
\hline $\begin{array}{l}\text { Identity of other player } \\
\text { P12: 'At this point, I'm starting to be a bit mad. Umm ... I don't know why. } \\
\text { They might be a woman. Might be. But I don't associate women with such } \\
\text { greediness.' }\end{array}$ & 1 & 2 \\
\hline
\end{tabular}

